\title{
Activity spread and breathers induced by finite transmission speeds in two-dimensional neural fields
}

\author{
Axel Hutt* and Nicolas Rougier \\ INRIA Nancy - Grand Est, Team CORTEX, 615 rue du Jardin Botanique, 54600 Villers-les-Nancy, France
}

(Dated: October 29, 2010)

\begin{abstract}
The work studies the spatio-temporal activity propagation in a two-dimensional spatial system involving a finite transmission speed. We derive a numerical scheme in detail to integrate the corresponding evolution equation and validate the derived algorithm by a study of a spatially periodic system. Finally, the work demonstrates numerically novel transmission delay-induced breathers subjected to anisotropic external input.
\end{abstract}

Finite transmission speed in physical systems has attracted research for decades. Previous work on heat diffusion has shown experimentally that the transmission speed (also called propagation speed in the literature) is finite in certain media $[1,2]$. These results do not show accordance to classical diffusion theory implying infinite transmission speed. To cope with this problem theoretically, Cattaneo was one of the first to insert delay terms into the diffusion equation to achieve a finite transmission speed [3].

Recently, an integral model has been proposed which takes into account a finite transmission speed as a space-dependent retardation [4]. It was shown that the Cattaneo-equation can be derived from this model. Interestingly, this model is well-established in computational neuroscience where it describes the activity evolution in a neural population involving finite transmission speed along axonal fibres. This so-called neural field model has been shown to model successfully neural activity known from experiments $[5,6]$. In the recent decades, neural fields have been studied analytically and numerically in one and two spatial dimensions [7, 8], while previous studies considered finite axonal transmission speeds in one-dimensional models only [9]. To our best knowledge, no previous study considered analytically and numerically finite transmission speeds in two-dimensional neural fields. The current work fills this gap to reveal finite transmission speed effects in two-dimensional systems. To this end, the subsequent paragraphs derive a novel fast numerical scheme to simulate the corresponding evolution equations. Then stimulus-induced activity propagation is studied numerically to illustrate the delayed activity spread. Moreover, we find numerically transmission delay-induced breathers which have not been reported before.

Let us assume a two-dimensional quadratic spatial domain $\Omega$ with side length $l$ and periodic boundary conditions. Then the neural population activity $V(\mathbf{x}, t)$, i.e. the mean membrane potential, at spatial location $\mathbf{x} \in \Omega$

\footnotetext{
*Axel.Hutt@loria.fr
}

and time $t$ obeys the evolution equation

$$
\begin{aligned}
& \tau \frac{\partial}{\partial t} V(\mathbf{x}, t)=I(\mathbf{x}, t)-V(\mathbf{x}, t) \\
& \quad+\int_{\Omega} d^{2} y K(|\mathbf{x}-\mathbf{y}|) S\left[V\left(\mathbf{y}, t-\frac{|\mathbf{x}-\mathbf{y}|}{c}\right)\right]
\end{aligned}
$$

with the synaptic time constant $\tau$, the external stimulus $I(\mathbf{x}, t)$, the finite axonal transmission speed $c$ and the nonlinear transfer function $S$. Moreover, the spatial interaction is non-local and is given by the spatial synaptic connectivity kernel $K(|\mathbf{x}-\mathbf{y}|)$, that depends on the distance between two spatial locations $\mathbf{x}$ and $\mathbf{y}$ only.

To investigate the activity propagation in detail, we derive a novel iteration scheme for the numerical integration of (1). Since the integral over space in (1) is not a convolution in the presence of a finite transmission speed $c$, one can not apply directly fast numerical algorithms such as the Discrete Fast Fourier Transform (DFT) to calculate the integral. Hence the numerical integration of (1) is very time consuming with standard quadrature techniques. For instance, with a discretization of the spatial domain by $N^{2}$ grid intervals and applying the Gaussian quadrature rule for the spatial integral, it would be necessary to compute $N^{4}$ elements in each time step what is very time-consuming in case of a good spatial resolution. The present work proposes a fast numerical method that is based on the DFT and resembles the Ritz-Galerkin method well-established to solve partial differential equations. To this end, we re-formulate the problem by introducing a delay kernel, that in turn allows to write the integral again as a convolution in space and time.

We extend the integral in (1) to

$$
\begin{aligned}
& A(\mathbf{x}, t)=\int_{\Omega} d^{2} y K(|\mathbf{x}-\mathbf{y}|) S\left[V\left(\mathbf{y}, t-\frac{|\mathbf{x}-\mathbf{y}|}{c}\right)\right] \\
= & \int_{\Omega} d^{2} y \int_{-\infty}^{\infty} d t^{\prime} L\left(\mathbf{x}-\mathbf{y}, t-t^{\prime}\right) S\left[V\left(\mathbf{y}, t^{\prime}\right)\right] \\
= & \int_{\Omega} d^{2} y \int_{0}^{\tau_{\max }} d \tau L(\mathbf{x}-\mathbf{y}, \tau) S[V(\mathbf{y}, t-\tau)],
\end{aligned}
$$

with $\tau_{\max }=l / \sqrt{2} c$, the novel spatial delay kernel $L(\mathbf{x}, \tau)=K(\mathbf{x}) \delta(|\mathbf{x}| / c-\tau)$ and the delta-distribution $\delta(\cdot)$. These simple calculations show that $A$ may be written as a spatial convolution, but with a new delayed 
spatio-temporal kernel $L$ that now considers the past activity. The form (2) has been used previously to study spatio-temporal instabilities in one- and two-dimensional neural fields [7].

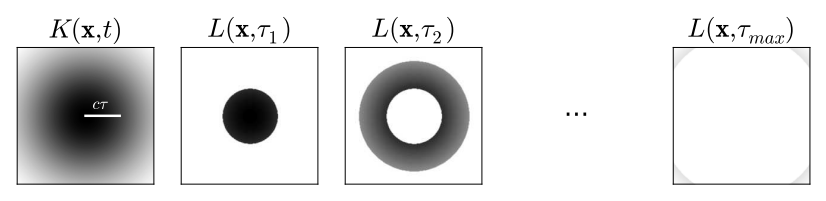

FIG. 1. The illustrative construction of the delay-kernel $L(\mathbf{x}, \tau)$. Assuming a spatial kernel (left image), $L$ exhibits rings with radius $c \tau$ (images on the right for different delay times) which is the interaction distance of the system at the delay time $\tau$.

We observe in (3) that the new delay kernel $L$ is independent of time $t$ and is computed on the delay interval only. Hence it represents the contribution of the current and past activity to the current activity at time $t$. In addition, we observe that $A$ implies multiple delays and the corresponding delay distribution function depends strongly on the spatial kernel $K$. In other words, axonal transmission speeds represent a distribution of delays, what has been found before in other contexts [10, 11]. Figure 1 illustrates the construction of the kernel: given the kernel function $K$ in space (Fig. 1, left image), $L(\mathbf{x}, \tau)$ is generated by cutting out a ring of radius $c \tau$ (Fig. 1, images on the right hand side). In a continuous spatial domain these rings are infinitely thin, whereas a spatially discretized domain yields finite ring widths, see the paragraphs below for more details.

Now let us derive the rules to compute $A$ numerically. The periodic boundary conditions lead to discrete wave vectors $\mathbf{k}_{m n}=\left(k_{m}, k_{n}\right)$ with $k_{p}=2 \pi p / l, p \in \mathcal{Z}_{0}$. Then $V(\mathbf{x}, t)$ may be expanded into the Fourier series

$$
V(x, y, t)=\frac{1}{l} \sum_{m, n=-\infty}^{\infty} \tilde{V}_{m n}(t) \mathrm{e}^{i\left(k_{m} x+k_{n} y\right)}
$$

with the Fourier vector component $\tilde{V}_{m n}(t)=$ $\tilde{V}\left(k_{m}, k_{n}, t\right)$. In addition, we find the spatial Fourier transform

$$
\tilde{V}_{m n}(t)=\frac{1}{l} \int_{-l / 2}^{l / 2} d x \int_{-l / 2}^{l / 2} d y V(x, y, t) \mathrm{e}^{-i\left(k_{m} x+k_{n} y\right)}
$$

Inserting (4) into (3) and applying (5), $A$ reads

$$
\begin{aligned}
A(x, y, t)= & \sum_{m, n=-\infty}^{\infty} \mathrm{e}^{i\left(k_{m} x+k_{n} y\right)} \\
& \times \int_{0}^{\tau_{\max }} d T \tilde{L}_{m n}(T) \tilde{S}_{m n}(t-T),
\end{aligned}
$$

where $\tilde{L}_{m n}(t), \tilde{S}_{m n}(t)$ are the spatial Fourier transforms of $L(\mathbf{x}, t)$ and the nonlinear functional $S[V(\mathbf{x}, t)]$, respec- tively. In addition, we find

$$
\begin{aligned}
\tilde{L}_{m n}(T)=\frac{c^{2}}{l} & \int_{-l / 2 c}^{l / 2 c} \int_{-l / 2 c}^{l / 2 c} \\
& \times \delta(|\boldsymbol{\tau}|-T) K(|c \boldsymbol{\tau}|) \mathrm{e}^{-i c \mathbf{k}_{m n} \boldsymbol{\tau}} d^{2} \boldsymbol{\tau} .
\end{aligned}
$$

After obtaining $A(x, y, t)$ in the Fourier space for a continuous spatial domain, now we discretize the spatial domain to gain the final numerical scheme. To this end, $\Omega$ is discretized in a regular spatial grid of $N \times N$ elements with grid interval $\Delta x=l / N$. Hence $x \rightarrow x_{n}=$ $n \Delta x, n=-N / 2, \ldots, N / 2-1$. By virtue of this discretization, we can approximate (6) and (7) by applying the rectangular rule $\int_{a}^{b} f(x) d x \approx \Delta x \sum_{n=-N / 2}^{N / 2-1} f\left(x_{n}\right)$. The error is $E<(b-a) \Delta x^{2} f^{\prime \prime}(\xi) / 24, a<\xi<b$ for twice-differentiable functions $f$, i.e. the rectangular rule is a good approximation for smooth functions and large enough $N$. The same holds true for the discretization of the time integral and Eq. (7) reads

$$
\tilde{L}_{m n}\left(T_{u}\right)=\frac{l}{N^{2} \Delta t} \sum_{k, p=-N / 2}^{N / 2} L_{k p}\left(T_{u}\right) \mathrm{e}^{-i 2 \pi(k m+n p) / N}
$$

with the discrete version of the delay kernel $L$

$$
L_{k p}\left(T_{u}\right)=\delta\left(\Delta \tau \sqrt{k^{2}+p^{2}}, T_{u}\right) K\left(\left|\mathbf{x}_{k p}\right|\right) .
$$

By virtue of the isotropy of the spatial interactions, in addition we find the simple relation $L_{k p}\left(T_{u}\right)=K(u c \Delta t)$, i.e. the width of the rings in Fig. 1 is $c \Delta t$. In these latter calculations, we introduced the time discretization $\tau_{k p}=(k, p) \Delta \tau, \Delta \tau=\Delta x / c, T \rightarrow T_{u}=u \Delta t$ and $\delta(\tau-T) \rightarrow \delta_{n u} / \Delta t$ for $\tau \rightarrow \tau_{n}$. The symbol $\delta(\cdot, \cdot)$ is identical to the Kronecker symbol and is introduced for notational convenience.

Although the relation $L_{k p}\left(T_{u}\right)=K(u c \Delta t)$ appears quite intuitive and elucidates the discrete ring structure of $L$, it does not give directly the condition which grid point $(k, p)$ belongs to which delay ring. This condition may be read off the Kronecker symbol: $u$ is an integer number and hence $\delta\left(\Delta \tau \sqrt{k^{2}+p^{2}}, T_{u}\right)=1$ if $\left[\Delta \tau \sqrt{k^{2}+p^{2}} / \Delta t\right]=u$ with the integer operation $[a]$ that cuts off the decimal numbers. Consequently the grid points $(k, p)$ that contribute to the delay time $T_{u}$ obey $u \leq \frac{\Delta x}{c \Delta t} \sqrt{k^{2}+p^{2}}<u+1, u=0,1,2, \ldots, u_{\max }$ with $u_{\max } \stackrel{c \Delta t}{=}\left[\tau_{\max } / \Delta t\right]$, i.e. they lie in a ring with inner and outer radius $(c \Delta t / \Delta x) u$ and $(c \Delta t / \Delta x)(u+1)$, respectively.

From the definition of $L_{k p}\left(T_{u}\right)$, we can derive some conditions on the numerical parameters. The width of the rings in Fig.1 is $\Delta r=c \Delta t / \Delta x$ spatial grid intervals. Consequently, the maximum radius of a ring is $l / \sqrt{2} \Delta x$ and hence the maximum transmission speed that can be implemented is $c_{\max }=l / \sqrt{2} \Delta t$. Since $c_{\max } \rightarrow \infty$ for $\Delta t \rightarrow 0$, the transmission speed $c>c_{\max }$ in the discrete scheme is equivalent to an infinite transmission speed in 
the analytical original model and the finiteness of $c_{\max }$ results from the time discretization. Moreover, $c \rightarrow c_{\max }$ yields $\tau_{\max } \rightarrow 0$, i.e. the transmission delay vanishes.

Another consequence of the spatial discretization is the finite number of spatial Fourier modes in contrast to the infinite number of modes in continuous domains. The maximum wave number is $k_{\max }=2 \pi / \Delta x$ and, by the definition of $\Delta x$, the number of Fourier modes is limited to $N$.

Now combining the latter results, Eq. (6) reads

$$
\begin{aligned}
A\left(x_{r}, y_{s}, t_{v}\right)= & \sum_{m, n=-N / 2}^{N / 2-1} \mathrm{e}^{i 2 \pi(m r+n s) / N} \\
& \times \sum_{u=0}^{u_{\max }-1} \tilde{L}_{m n}\left(T_{u}\right) \tilde{S}_{m n}\left(t_{v}-T_{u}\right) .
\end{aligned}
$$

Utilizing the standard definition of the twodimensional Discrete Fourier Transform $D F T[A]_{k p}=$ $\sum_{n, m} A_{n m} \exp (-i 2 \pi(n k+m p) / N), \quad n, m \in$ $[-N / 2 ; N / 2-1]$ and its inverse (IDFT) correspondingly, we find finally

$$
\begin{aligned}
A\left(\mathbf{x}, t_{v}\right)= & \frac{l^{2}}{N^{2}} \operatorname{IDFT}[ \\
& \left.\sum_{u=0}^{u_{\max }-1} \operatorname{DFT}\left[L\left(T_{u}\right)\right] \times \operatorname{DFT}\left[S\left(t_{v}-T_{u}\right)\right]\right] .
\end{aligned}
$$

Some numerical implementations of the DFT assume that the index $n$ runs in the interval $[0 ; N-1]$. In this case, Eq. (10) is also valid but $D F T[A]_{k p}$ is modulated by a factor $e^{-i \pi(k+p)}=(-1)^{k+p}$.

In practice, $D F T\left[L\left(T_{u}\right)\right]$ is computed once for all $T_{u}$ in the beginning of the simulation since it does not depend on the system activity. Moreover, for $N=2^{n}, n \in \mathcal{N}$, the discrete Fourier Transform may be implemented numerically by a Fast Fourier transform, that speeds up the numerical computation dramatically. This possible algorithm choice represents the major advantage of the proposed method compared to other non-convolution methods.

The discrete version of $A$ can be applied to any explicit or implicit numerical integration scheme. For instance, the numerical Euler scheme stipulates

$$
\begin{aligned}
& \tilde{V}_{m n}\left(t_{i}+\Delta t\right)=\tilde{V}_{m n}\left(t_{i}\right)+\frac{\Delta t}{\tau}\left(I_{m n}\left(t_{i}\right)-\tilde{V}_{m n}\left(t_{i}\right)\right. \\
& \left.+\frac{L^{3}}{N^{4}} \sum_{u=0}^{d} D F T\left[L\left(t_{u}\right)\right]_{m n} \times D F T\left[S\left(t_{v}-t_{u}\right)\right]_{m n}\right)
\end{aligned}
$$

where $I_{m n}(t)$ is the Fourier transform of the input $I(\mathbf{x}, t)$ and one obtains $V\left(\mathbf{x}, t_{v}\right)$ by applying Eq. (4).

In the following, we study analytically and numerically the response to an external stimulus. At first, let us consider a small input. Then the response is linear about the systems' stationary state. Since we are interested in responses that approach the stationary state after removal of the stimulus, it is necessary to ensure the linear stability of the stationary state.

The stationary state of Eq. (1) constant in space and time implies $V_{0}=\kappa S\left[V_{0}\right]+I_{0}$ for a constant input $I_{0}$ with the kernel norm $\kappa=\int_{\Omega} K(\mathbf{x}) d^{2} x$. Considering small additional external inputs $\bar{I}(\mathbf{x}, t)=I(\mathbf{x}, t)-I_{0}$, small deviations $u(\mathbf{x}, t)=V(\mathbf{x}, t)-V_{0}$ from this stationary state obey

$$
\begin{aligned}
\frac{d u(\mathbf{x}, t)}{d t}= & -u(\mathbf{x}, t)+\bar{I}(\mathbf{x}, t) \\
& +s^{\prime} \int_{\Omega} K(\mathbf{x}-\mathbf{y}) u(\mathbf{y}, t-|\mathbf{x}-\mathbf{y}| / c) d y^{2}(.12)
\end{aligned}
$$

with $s^{\prime}=\delta S[V] / \delta V, V=V_{0}$. Now expanding $u(\mathbf{x}, t)$ into a spatial Fourier series according to Eq. (4) and applying a temporal Laplace transform to each Fourier mode amplitude, we find the characteristic equation

$$
\lambda+1=\int_{\Omega} K(\mathbf{x}) e^{i \mathbf{k x}-\lambda|\mathbf{x}| / c} d^{2} x
$$

with the wave vector $\mathbf{k}=\left(k_{m}, k_{n}\right)^{t}$ and the Lyapunov exponent $\lambda \in \mathcal{C}$. The stationary state $V_{0}$ is linearly stable if $\operatorname{Re}(\lambda)<0$.

Now let us consider the spatio-temporal response of the system involving the spatially periodic interactions $K(\mathbf{x})=K_{o} \sum_{i=0}^{2} \cos \left(\mathbf{k}_{i} \mathbf{x}\right) \exp (-|\mathbf{x}| / \sigma)$ with $\mathbf{k}_{i}=$ $k_{c}\left(\cos \left(\phi_{i}\right), \sin \left(\phi_{i}\right)\right)^{t}, \phi_{i}=i \pi / 3$. This kernel reflects spatial hexagonal connections which have been found, e.g., in layer $2 / 3$ of the visual cortex in monkeys [12]. Stimulating the stable system by a small external input in the presence of the finite transmission speed $c$ elucidates the transmission delay effect on the linear response. This delay effect has attracted some attention in previous studies on the activity propagation in the visual cortex $[13,14]$. For the given kernel, the characteristic equation (13) reads $\lambda+1=\sum_{i=0}^{2} f_{+}\left(\lambda, \phi_{i}\right)+f_{-}\left(\lambda, \phi_{i}\right)$ with $f_{ \pm}\left(\lambda, \phi_{i}\right)=$ $1 /{\sqrt{(1 / \sigma+\lambda / c)^{2}+k^{2}+k_{c}^{2} \pm 2 k k_{c} \cos \left(\phi_{i}-\theta\right)}}^{3}$ and $\mathbf{k}=$ $k(\cos (\theta), \sin (\theta))^{t}$. The numerical simulation applies parameters which guarantee the stability of the stationary state.

Figure 2 shows snapshots of the simulated spatiotemporal response of the system about a stable stationary state applying the numerical scheme (11). We observe the lateral activity propagation starting from the stimulus location in the domain centre. The spreading activity reveals the maxima of axonal connections close to previous experimental findings [12]. To validate the numerical results, we take a closer look to two single spatial locations, denoted $A$ and $B$ in Fig. 2 at distance $d_{A}$ and $d_{B}$ from the stimulus location in the center. Before stimulus onset, they show the stationary activity constant in time. After stimulus onset, it takes the activity some time to propagate from the stimulus location to these distant points, i.e. the transmission delays $d_{A} / c=2.1 \mathrm{~ms}$ and $d_{B} / c=3.8 \mathrm{~ms}$ with the transmission speed $c$. In 
fact, Fig. 2 shows that the activity reaches the locations $A$ and $B$ about these times for the first time as expected. This finding validates the numerical algorithm proposed above. Moreover, the chosen parameters are realistic for layers $2 / 3$ of the visual cortex and hence the results elucidates that the axonal transmission delay is important to understand the activity spread in the visual cortex as pointed out in previous studies, e.g. [14].


FIG. 2. Spatio-temporal response activity to the external stimulus $I(\mathbf{x}, t)=I_{0}+e^{-\mathbf{x}^{2} / \sigma_{I}^{2}}$ for the spatial connectivity function $K(\mathbf{x})$ by the numerical simulation of Eq. (1). Used (dimensionless) parameters are $K_{o}=0.1, c=10, l=$ $10, k_{c}=10 \pi / l, \sigma=10, \sigma_{I}=0.2, N=512, \tau=1, \Delta t=0.005$. Moreover, $I_{0}=2.0, S[V]=2 /(1+\exp (-5.5(V-3)))$ and $V_{0}=2.00083$. The initial values are chosen to $V(\mathbf{x}, \theta)=V_{0}$ for the delay interval $-l / \sqrt{2} c \leq \theta \leq 0$. Introducing the temporal and spatial scale scale $\tau=10 \mathrm{~ms}$ and $\lambda=1.0 \mathrm{~mm}$, the results reflect the spatio-temporal activity with transmission speed $c=1.0 \mathrm{~m} / \mathrm{s}$ and the domain length $l=10 \mathrm{~mm}$, which are realistic values for layer $2 / 3$ in visual cortex. Then the points $A$ and $B$ are located at a distance $d_{A}=2.1 \mathrm{~mm}$ and $d_{B}=3.8 \mathrm{~mm}$ from the stimulus onset location at the origin, respectively. The bar in the plots is $0.83 \mathrm{~mm}$ long.

Finally, we ask whether the transmission delay may induce oscillatory instabilities in the presence of external input. The following brief numerical study is motivated by previous theoretical studies on breathers [15]. In that study, the authors computed analytically conditions for Hopf-bifurcations from stimulus-induced stable standing bumps in a neural model involving spike rate adaption. The corresponding control parameter of these instability studies is the magnitude of the applied external stimulus. In contrast, the present model does not consider spike rate adaption and decreases the axonal transmission speed, i.e. increases the transmission delay, to evoke a delay-induced Hopf-bifurcation while keeping the other parameters constant. Since an analytical treat-

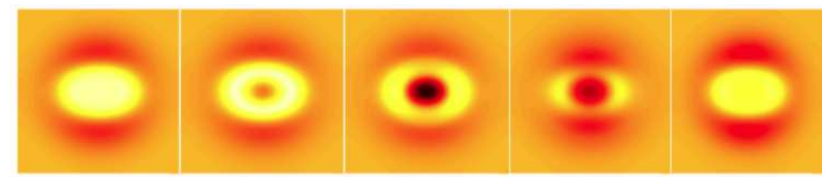

FIG. 3. (Color online) Transmission delay-induced breathers evoked by an anisotropic external stimulus. The spatial connectivity function is chosen to $K(r)=10 \exp (-r / 3) /(18 \pi)-$ $14 \exp (-r / 7) /(98 \pi)$ and the input magnitude and variances are $I_{0}=10$ and $\Sigma_{11}^{-1}=3, \Sigma_{22}^{-1}=5$, respectively. Further parameters are $c=100, l=30, N=512, \tau=1, \Delta t=0.05$ and $V_{\text {thresh }}=0.005$. The initial values are chosen to $V(\mathbf{x}, \theta)=0$ for the delay interval $-L / \sqrt{2} c \leq \theta \leq 0$.

ment would exceed the aim of the current rapid communication by far, we just apply a numerical study to show this novel effect. We apply an anisotropic Gaussian stimulus $I(\mathbf{x}, t)=I_{0} e^{-\mathbf{x}^{t} \boldsymbol{\Sigma}^{-1} \mathbf{x} / 2}$ with the $2 \times 2$ diagonal variance matrix $\Sigma^{-1}$ with $\Sigma_{i i}^{-1}=1 / \sigma_{i}^{2}, i=1,2$. Moreover the spatial kernel $K(\mathbf{x})$ represents locally excitatory and laterally inhibitory connections and the transfer function is the Heaviside function $S[V]=H\left[V-V_{\text {thresh }}\right]$. The numerical computation of Eq. (1) applying the numerical scheme (11) yields delay-induced breathers in two dimensions. Figure 3 shows the temporal sequence of a single oscillation cycle. To our best knowledge such delay-induced breathers in two dimensions have not been found before.

Concluding, we have derived analytically and validated numerically a novel numerical scheme for twodimensional neural fields involving transmission delay that includes a convolution structure and hence allows the implementation of fast numerical algorithms, such as Fast Fourier Transform. Moreover, we have demonstrated numerically a transmission delay-induced breather that has not been observed before. To facilitate future applications of the algorithm, the implementation code for both numerical examples is made available for download [16]. In future research, the transmission delay will play an important role in the understanding of fast activity propagations whose time scales are close to the transmission delay, e.g. in the presence of ultra-fast pulses and/or at spatial nanometer scales.
[1] E. Lazzaro and H. Wilhelmsson, Phys. Plasmas, 5, 2830 (1998).

[2] J. Klossika et al., Phys.Rev.B, 54, 10277 (1996).

[3] C. Cattaneo, Comptes Rendues, 247, 431 (1958).

[4] A. Hutt, Phys. Rev. E, 75, 026214 (2007).

[5] X. Huang et al., J.Neurosc., 24, 9897 (2004).

[6] P. C. Bressloff et al., Neural Comput., 14, 473 (2002).

[7] N. Venkov et al., Physica D, 232, 1 (2007); S. Coombes et al., Phys. Rev. E, 76, 051901 (2007).

[8] C. Laing, SIAM J. Appl. Dyn. Syst., 4, 588 (2005); M. R. Owen et al., New J. Phys., 9, 378 (2007); N. Rougier, J. Vitay, Neur. Networks, 19, 773-581 (2006).
[9] S. Coombes, Biol. Cybern., 93, 91 (2005).

[10] A. Hutt and T. Frank, Acta Phys. Pol. A, 108, 1021 (2005).

[11] G. Faye and O. Faugeras, Physica D, 239, 561 (2010).

[12] J. Lund et al., Cerebral Cortex, 13, 15 (2003).

[13] L. Schwabe et al., J.Neurosci., 26, 9117 (2006).

[14] V. Bringuier et al., Science, 283, 695 (1999).

[15] S. Folias and P. Bressloff, Phys. Rev. Lett., 95, 208107 (2005).

[16] N. Rougier and A. Hutt, http://www.loria.fr/ rougier/research/DNF.html. 